\title{
Chronic Cough and Eosinophilic Esophagitis: An Uncommon Association
}

\author{
Paolo Orizio $^{a}$ Massimo Cinquini ${ }^{b}$ Stefano Minettib \\ Daniele Alberti ${ }^{\mathrm{a}}$ Camilla Di Paolo $^{\mathrm{b}}$ Vincenzo Villanacci ${ }^{\mathrm{c}}$

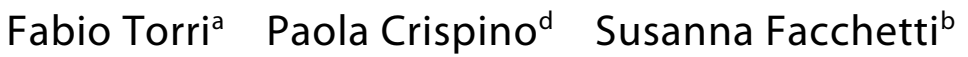 \\ Fabio Lodi Rizzini $^{b}$ Gabrio Bassotti ${ }^{\mathrm{e}}$ Cinzia Tosoni ${ }^{b}$
}

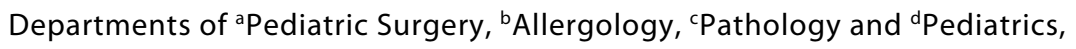
Spedali Civili, Brescia, and 'Department of Clinical and Experimental Medicine, University of Perugia, Italy

\section{Key Words}

Eosinophilic esophagitis · Gastroesophageal reflux disease $\cdot$ Chronic cough

\begin{abstract}
An increasing number of children, usually with gastrointestinal symptoms, is diagnosed with eosinophilic esophagitis $(\mathrm{EE})$, and a particular subset of these patients complains of airway manifestations. We present the case of a 2-year-old child with chronic dry cough in whom EE was found after a first diagnosis of gastroesophageal reflux disease (GERD) due to pathological 24-hour esophageal pH monitoring. Traditional allergologic tests were negative, while patch tests were diagnostic for cow's milk allergy. We discuss the intriguing relationship between GERD and EE and the use of patch test for the allergologic screening of patients.
\end{abstract}

\section{Introduction}

Eosinophilic esophagitis (EE) is defined as a primary clinicopathologic disorder of the esophagus characterized by esophageal and/or upper gastrointestinal tract symptoms [1], whose clinical findings in adults include food impaction and dysphagia, whereas feeding intolerance and symptoms consistent with gastroesophageal reflux disease (GERD) are more frequently observed in children [2]. The key histological finding is an eosinophilic infiltration of the esophageal mucosa with more than 15 intraepithelial eosinophils/ high-power field (HPF) in one or more biopsies, and a dense esophageal eosinophilia is often described in association with severe squamous epithelial hyperplasia, whereas the 
gastric and duodenal mucosae are spared [2]. Moreover, a diagnostic requirement is the absence of GERD, which can be assessed with an esophageal $\mathrm{pH}$ monitoring study or after a high-dose proton pump inhibitor (PPI) treatment [1]. The correlation between EE, atopy, aeroallergen polysensitization and food allergens has been well documented [3]; indeed, a correlation between food allergens and EE was first demonstrated by Kelly et al. who observed that an elemental diet improved clinical and histological findings in 10 children with EE [4]. These findings were subsequently confirmed by other authors.

Although an increasing number of children and adults are diagnosed as suffering from EE, only few controlled trials have been performed to guide clinical management; thus, clinical practice is largely based on limited data and experts' opinions [2]. Here we report the case of a 2-year-old child who was diagnosed with EE with an atypical clinical presentation and an unusual diagnostic course.

\section{Case Report}

A 2-year-old child was referred to the Pediatric Surgical Department of Spedali Civili of Brescia complaining of dry cough with nocturnal exacerbations in the last 6 months, associated with retching and failure to thrive. She had previously been diagnosed with atopic dermatitis and persistent rhinoconjunctivitis; peripheral eosinophil count was normal. Due to a suspicion of GERD, 24-hour esophageal $\mathrm{pH}$ monitoring was performed resulting in a pathologic reflux index (12\%), and a 3-month high-dose treatment with PPI (esomeprazole $2 \mathrm{mg} / \mathrm{kg} /$ day) was prescribed, without clinical improvement. An esophagogastroduodenoscopy (EGDS) was carried out; esophageal linear furrows were observed and an eosinophilic infiltration of the esophageal mucosa ( $>20$ eosinophils/HPF) was evident at histology (fig. 1a-c), whereas gastric and duodenal findings were endoscopically and histologically normal. On these grounds the possibility of EE was raised, and the patient was referred to the Allergy Unit of this hospital. Perennial and seasonal aeroallergens and food-specific serum IgE (CAP System, Phadia, Uppsala, Sweden) were absent and skin prick tests for common aeroallergens were negative. Since EE in children is often associated with food allergy, and although in vitro tests with commercially available food extracts were negative, prick to prick skin tests with fresh foods were performed. However, only a very weak sensitization to tomato was detected and Solanaceae exclusion from the diet did not result in any clinical improvement. Nevertheless, since acute episodes appeared closely related to food intake and since milk and egg are reported among the main causative food in EE [5], a restricted dietary regimen was implemented, withdrawing milk and eggs. Clinical improvement was observed after 1 week when the cough completely disappeared. After 2 weeks of dietary restriction the patient was accidentally exposed to milk proteins as she was fed with sliced carrots that had been prepared with a cheese grater. Recurrence of cough was reported about $3 \mathrm{~h}$ after the meal. After 1 month the patient gained weight and reported a steady and complete resolution of all symptoms, including cough. Egg intake was not followed by clinical relapse, whereas milk dietary reintroduction was no longer attempted.

In order to confirm an etiopathogenetic correlation between cow's milk sensitization and EE, patch tests with cow's milk proteins were applied on the forearm. The tests were performed mixing $2 \%$ skin prick test commercial extract (Lofarma) to vaseline. Beta-lactoalbumin, lactoglobulin and casein were tested. After 48-hour occlusion, an infiltrated erythematous papula developed at the casein application site. As a result, a long-term restricted diet with no milk or derivates was prescribed. Two months later, with the patient completely asymptomatic, an EGDS with esophageal biopsies was repeated and showed normal-appearing mucosa; the eosinophilic esophageal infiltration was no longer detectable (fig. 1d-f). Esophageal pH monitoring displayed normal values (reflux index 4.5\%). Consequently the diagnosis of EE due to milk allergy was confirmed. After 4 months, the patients ate an ice cream made of cow's milk and persistent intense coughing appeared $3 \mathrm{~h}$ later. On that occasion no therapeutic measures were taken but cow's milk was strictly avoided thereafter. A follow-up visit was performed 2 years after the diagnosis and the patient reported being completely asymptomatic. 


\section{Discussion}

Dry cough with nocturnal exacerbations is a symptom described in GERD, usually defined as an atypical manifestation of the condition, but it is not generally found in EE patients, even though a particular subset of patients has emerged with airway manifestations [6]. The relationship between GERD and EE is not always still, as EE diagnosis requires exclusion of pathologic acid reflux with a PPI trial and/or a normal esophageal $\mathrm{pH}$ monitoring [2], but pathologic acid reflux in EE is described by some authors in a significant proportion of patients [7]. On the other hand, eosinophilic infiltration of esophageal mucosa is well described in GERD [8]; thus, the mere finding of a pathologic esophageal $\mathrm{pH}$ test cannot rule out $\mathrm{EE}$, and the mere presence of an eosinophilic infiltration is not diagnostic of EE. The diagnostic guidelines developed by gastroenterologists are based on biopsy specimen findings of $\geq 15$ eosinophils/HPF [2], but the maximal number of eosinophils/HPF that is associated with GERD-related esophagitis is still under investigation. Brigger et al. [9] underlined that the distinction between EE and GERD cannot be reliably performed on histopathologic evidence alone in children with upper aerodigestive symptoms.

In our patient the symptom and the result of the $\mathrm{pH}$ study led us to suspect GERD and to start treatment with PPI. After a 3-month trial without clinical improvement an EGDS was advised and EE detected. A possible explanation for the abnormal esophageal acid exposure could be due to a esophageal motility disorder and/or to lower esophageal sphincter incompetence, possibly associated to an inflammatory infiltration, but the literature is discordant about this topic [10]. We had a definite diagnosis only after a restricted dietary regimen with resolution of symptoms and of esophageal inflammatory infiltration.

In GERD chronic cough is considered the result of acid stimulation of esophageal chemoreceptors or the effect of direct acid irritation of laryngeal mucosa. In our patient acidity was not implicated as PPI therapy did not have significant effects. We feel that esophageal eosinophilic inflammation was allergen-mediated (milk protein) rather than acid-mediated. In general, eosinophils have proinflammatory effects, upregulating adhesion systems, mediating cell trafficking, and releasing cytokines, chemokines, lipid mediators and effector proteins. More in depth, eosinophil cationic proteins participate in tissue damage eventually resulting in fibrosis [11]. Eosinophil major basic protein, the predominant toxic protein found in eosinophilic granules, has been established as a critical cytotoxin in the pathophysiology of asthma and chronic rhinosinusitis [12]. This protein directly induces mast cell degranulation, airway reactivity and smooth muscle contraction, and increases smooth muscle responsiveness to agonist [13]. Deposition of major basic protein in the esophageal mucosa in patients with EE has been described [14], although its role in the pathophysiology of this condition has not been explored.

In our patient there might have been an initial esophageal sensitization to milk proteins, with EE occurring on esophageal re-challenge with the same antigen. In this scenario the cough may result from the aforementioned eosinophilic infiltration. Alternatively, Mishra et al. [15] showed a link between allergic hypersensitivity response in the lung and the esophagus, but to our knowledge there are no previous studies showing a simultaneous esophageal and pulmonary eosinophilia due to food allergen exposure. Furthermore in our case only a cell-mediated mechanism was detected. Indeed, since most patients with EE display positive reactions to foods on skin prick test and/or 


\begin{tabular}{r|l|l|l} 
Case Reports in & Case Rep Gastroenterol 2011;5:497-501 & $\begin{array}{l}\text { Published online: } \\
\text { August 27, 2011 }\end{array}$ & $\begin{array}{l}\text { O 2011 S. Karger AG, Basel } \\
\text { ISSN 1662-6031 } \\
\text { www.karger.com/crg }\end{array}$ \\
\hline Gastroenterology
\end{tabular}

atopy patch test, a mixed immunopathogenesis, both IgE- and cell-mediated, has been postulated [5]. A traditional allergologic screening only based on in vitro and percutaneous tests would not have been sufficient to identify the responsible food allergen.
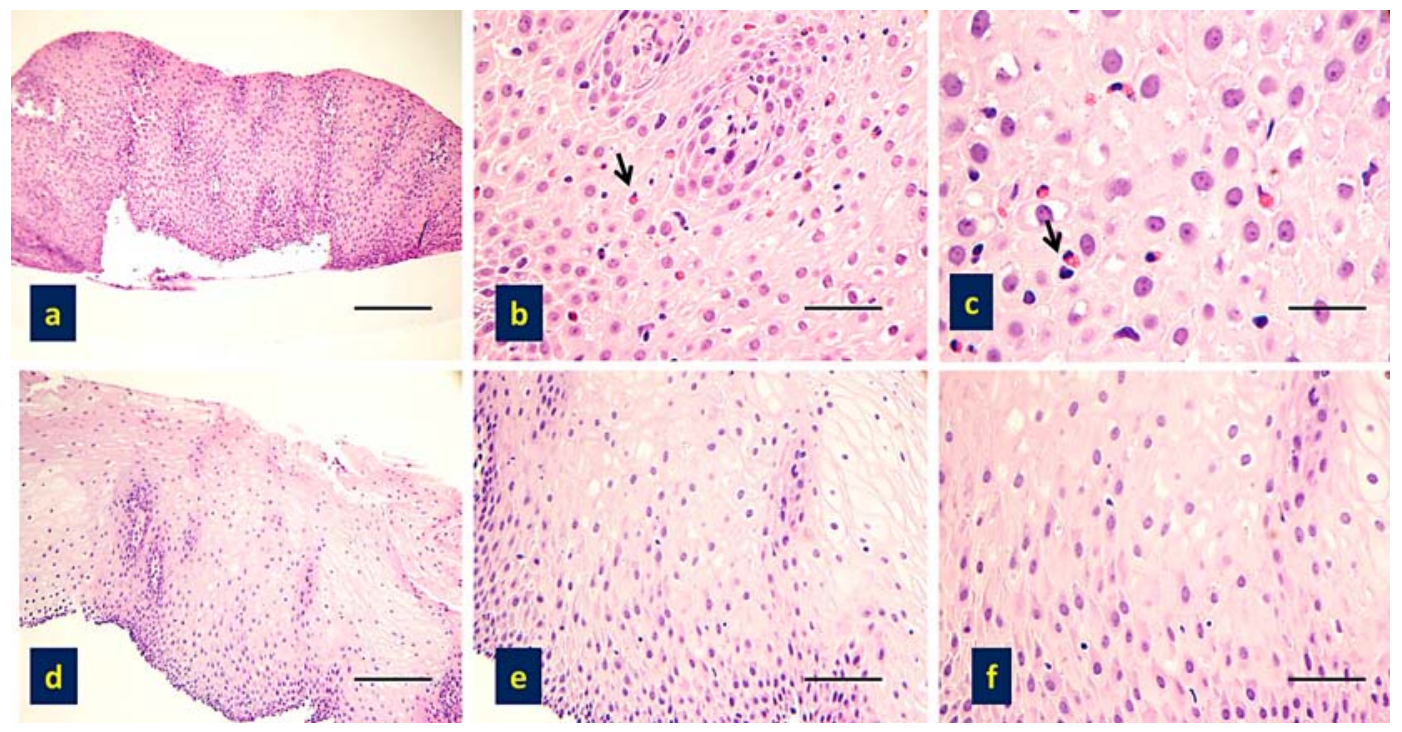

Fig. 1. a-c Basal histological specimens of the child showing the presence of EE, as demonstrated by a pathological increase of eosinophils (arrows) in the esophageal mucosa. $\mathrm{H} \& \mathrm{E}$, scale bars $=100 \mu \mathrm{m}$. d-f Histological sampling of the child after diet, showing complete absence of eosinophils in the esophageal mucosa. $\mathrm{H} \& \mathrm{E}$, scale bars $=100 \mu \mathrm{m}$.

\section{References}

- Gupte A, Draganov P: Eosinophilic esophagitis. World J Gastroenterol 2009;15:17-24.

2 Furuta GT, Liacouras CA, Collins MH, et al: Eosinophilic esophagitis in children and adults: a systematic review and consensus recommendations for diagnosis and treatment. Gastroenterology 2007;133:1342-1363.

3 Roy-Ghanta S, Larosa DF, Katzka DA: Atopic characteristics of adult patients with eosinophilic esophagitis. Clin Gastroenterol Hepatol 2008;6:531-535.

4 Kelly KJ, Lazenby AJ, Rowe PC, et al: Eosinophilic esophagitis attributed to gastroesophageal reflux: improvement with an amino acid-based formula. Gastroenterology 1995;109:1503-1512.

5 Spergel JM, Andrews T, Brown-Whitehorn TF, et al: Treatment of eosinophilic esophagitis with specific food elimination diet directed by a combination of skin prick and patch tests. Ann Allergy Asthma Immunol 2005;95:336-343.

6 Orenstein SR, Shalaby TM, Di Lorenzo C, et al: The spectrum of pediatric eosinophilic esophagitis beyond infancy: a clinical series of 30 children. Am J Gastroenterol 2000;95:1422-1430.

7 Remedios M, Campbell C, Jones DM, et al: Eosinophilic esophagitis in adults: clinical, endoscopic, histologic findings and response to treatment with fluticasone propionate. Gastrointest Endosc 2006;63:3-12.

8 Ahmad M, Soetikno RM, Ahmed A: The differential diagnosis of eosinophilic esophagitis. J Clin Gastroenterol 2000;30:242-244. 
9 Brigger MT, Misdraji J, Hardy SC, et al: Eosinophilic esophagitis in children: a pathologic or clinicopathologic diagnosis? Arch Otolaryngol Head Neck Surg 2009;135:95-100.

10 Sant'Anna AM, Rolland S, Fournet JC, et al: Eosinophilic esophagitis in children: symptoms, histology, and pH probe results. J Pediatr Gastroenterol Nutr 2004;39:373-377.

-11 Rothenberg ME, Mishra A, Brandt EB, et al: Gastrointestinal eosinophils. Immunol Rev 2001;179:139-155.

12 Gundel RH, Letts LG, Gleich GJ: Human eosinophil major basic protein induces airway constriction and airway hyperresponsiveness in primates. J Clin Invest 1991;87:1470-1473.

13 Flavahan NA, Slifman NR, Gleich GJ, et al: Human eosinophil major basic protein causes hyperreactivity of respiratory smooth muscle. Role of the epithelium. Am Rev Respir Dis 1988;138:685-688.

14 Furuta GT, Walsh S, Antonioli DA, et al: Eosinophil major basic protein deposition occurs in eosinophilic esophagitis (abstract). Gastroenterology 1998;114:G0518.

15 Mishra A, Hogan SP, Brandt EB, et al: An etiological role for aeroallergens and eosinophils in experimental esophagitis. J Clin Invest 2001;107:83-90. 